\title{
Foreword
}

The Twenty-third International Congress of Onomastic Sciences was held at York University in Toronto, August 17-22, 2008. This was the second time the Congress was held in Canada, the previous occasion being in 1987 at Laval University in Quebec City, and the third time in North America, including the meeting at the University of Michigan at Ann Arbor, U.S.A. in 1981.

The general theme of the Congress was Names in Contact: Names in a Multi-Lingual, MultiCultural, Multi-Ethnic World, an appropriate topic for Toronto, the most multi-ethnic city in North America, and reportedly in the world. The Congress was well attended with some 180 participants from 37 countries. 160 papers were read on a wide variety of topics focusing on the general theme of the meeting, illustrating the diverse and far-reaching nature of onomastics as a discipline. These Proceedings contain 118 papers that have been reviewed by the Selection Committee. While the theme of the Congress constitutes the general background of most contributions, the papers address an extraordinarily rich mosaic of topics in toponymy, anthroponymy, brand names, etc. and involve a wide range of related disciplines such as history, geography, psychology, linguistics and several other areas of the humanities and social sciences.

These Proceedings proudly join the Proceedings that were published by most of the previous twenty-two conferences of the International Council of Onomastic Sciences that have been held throughout Europe and North America since the 1937 founding conference in Paris. It is our hope that this set of papers will serve as a memorial to the 2008 meeting in Toronto as well as an invitation to future scholars to join a dynamic international interdisciplinary research community.

\section{Avant- propos}

Le vingt-troisième Congrès international des sciences onomastiques a eu lieu à l'Université York de Toronto, du 17 au 22 août 2008. Il s'agissait de la deuxième fois que le congrès avait lieu au Canada, la première occasion étant en 1987 à l'Université Laval à Québec et de la troisième fois en Amérique avec la rencontre à l’Université du Michigan aux États-Unis en 1981.

Le thème général du congrès était Les noms propres en contact : les noms propres dans un monde multilingue, multiculturel et multiethnique, une thématique appropriée pour Toronto, reconnue supposément comme la ville la plus multiethnique au monde. Le congrès a connu une forte participation avec quelque 180 congressistes en provenance de 37 pays. 160 communications ont été présentées sur une variété de sujets portant sur la thématique du congrès et illustrant la nature diversifiée ainsi que la portée considérable de l'onomastique en que discipline. Ces actes contiennent 118 communications qui ont été retenues par le Comité de Sélection. Bien que le thème du congrès constitue la toile de fond de la plupart des contributions, les articles portent sur une mosaïque extraordinairement riche de sujets en toponymie, anthroponymie, noms de marques de commerce, etc., et se rapportent à une large gamme de disciplines comme l'histoire, la géographie, la psychologie, la linguistique et plusieurs autres domaines des humanités et des sciences sociales.

Ces actes s'ajoutent fièrement à la liste des actes qui ont été publiés à la suite de la plupart des vingt-deux congrès précédents du Conseil international des sciences onomastiques qui ont eu lieu à travers l'Europe et l'Amérique du Nord depuis le congrès 
inaugural en 1937 à Paris. Nous espérons que cet ensemble de contributions servira de mémorial au congrès de 2008 et constituera en même temps une invitation aux chercheurs futurs à se joindre à une dynamique communauté internationale de recherche interdisciplinaire.

\section{Vorwort}

Der 23. Kongress für Namenkunde fand vom 17. bis zum 22. August 2008 an der York Universität in Toronto statt. Dies war das zweite Mal, dass der Kongress in Kanada abgehalten wurde. Das erste Mal war der Veranstaltungsort die Laval Universität in Quebec City. Es ist auch das dritte Mal, dass der Kongress in Nordamerika stattfand, wenn man den Kongress an der Universität Michigan zu Ann Arbor in den Vereinigten Staaten in Betracht zieht.

Das allgemeine Thema des Kongresses war Namen im Kontakt: Namen in einer vielsprachigen, multi-kulturellen und multi-ethnischen Welt. Dies ist ein angemessenes Thema für Toronto, die multi-ethnischste Stadt in Nordamerika, und vielleicht sogar auch in der ganzen Welt. Der Kongress erreichte eine höhere Teilnehmerzahl, ungefähr 180 Teilnehmer aus 37. Ländern. Es wurden 160 Vorträge gehalten, die sich hauptsächlich mit dem allgemeinen Thema des Kongresses befassten und damit das vielfältige und weitreichende Wesen der Onomastik darstellten. Die Sitzungsberichte enthalten 118 Vorträge, die von dem Auswahlkomittee rezensiert und überarbeitet wurden. Die meisten Beiträge befassen sich mit dem allgemeinen Thema des Kongresses, aber die Beiträge zeigen auch ein äußerst reiches Mosaik von Themen aus der Toponymie und der Anthroponymie, von Geschäftsnamen, usw., und sie beziehen sich auf verschiedene Wissenschaftsbereiche, z. B. Geschichte, Geographie, Psychologie, Linguistik und verschiendene andere Gebiete der Human- und Gesellschaftswissenschaften.

Diese Sitzungsberichte nehmen ihren Platz in der Reihe von den Sitzungsberichten, die von den meisten der vorhergehenden zweizwanzig Kongressen veröffentlicht wurden, Kongresse die in vielen Ländern Europas und auch in Nordamerika seit der Gründungskonferenz im Jahre 1937 in Paris stattfanden. Wir hoffen, dass diese Beiträge ein Andenken an das Treffen von 2008 darstellt und auch als Einladung für zukünftige Wissenschaftler dient, einem internationalen und interdisziplinären Wissenschaftlerbund beizutreten.

The Editors / Le comité editorial / Die Herausgeber, Wolfgang Ahrens, York University / Université York Sheila Embleton, York University / Université York André Lapierre, University of Ottawa / Université d'Ottawa 